\title{
Development of patient-specific 3D-printed breast phantom using silicone and peanut oils for magnetic resonance imaging
}

\author{
Rooa Sindi ${ }^{1,2}$, Yin How Wong ${ }^{3}$, Chai Hong Yeong ${ }^{3}$, Zhonghua Sun $^{1}$ \\ ${ }^{1}$ Discipline of Medical Radiation Sciences, School of Molecular and Life Sciences, Curtin University, Perth, WA, Australia; ${ }^{2}$ Radio-diagnostic and \\ Medical Imaging Department, Medical Physics Section, King Fahd Armed Forces Hospital, Jeddah, Kingdom of Saudi Arabia; ${ }^{3}$ School of Medicine, \\ Faculty of Health and Medical Sciences, Taylor's University, Subang Jaya, Selangor, Malaysia
}

Correspondence to: Zhonghua Sun. Professor, Discipline of Medical Radiation Sciences, School of Molecular and Life Sciences, Curtin University, Perth, WA 6845, Australia. Email: z.sun@curtin.edu.au.

Background: Despite increasing reports of 3D printing in medical applications, the use of 3D printing in
breast imaging is limited, thus, personalized 3D-printed breast model could be a novel approach to overcome
current limitations in utilizing breast magnetic resonance imaging (MRI) for quantitative assessment of
breast density. The aim of this study is to develop a patient-specific 3D-printed breast phantom and to
identify the most appropriate materials for simulating the MR imaging characteristics of fibroglandular and
adipose tissues. Methods: A patient-specific 3D-printed breast model was generated using 3D-printing techniques for the construction of the hollow skin and fibroglandular region shells. Then, the T1 relaxation times of the five selected materials (agarose gel, silicone rubber with/without fish oil, silicone oil, and peanut oil) were measured on a $3 \mathrm{~T}$ MRI system to determine the appropriate ones to represent the MR imaging characteristics of fibroglandular and adipose tissues. Results were then compared to the reference values of T1 relaxation times of the corresponding tissues: $1,324.42 \pm 167.63$ and $449.27 \pm 26.09 \mathrm{~ms}$, respectively. Finally, the materials that matched the $\mathrm{T} 1$ relaxation times of the respective tissues were used to fill the $3 \mathrm{D}$-printed hollow breast shells.

Results: The silicone and peanut oils were found to closely resemble the T1 relaxation times and imaging characteristics of these two tissues, which are 1,515.8 \pm 105.5 and $405.4 \pm 15.1 \mathrm{~ms}$, respectively. The agarose gel with different concentrations, ranging from 0.5 to $2.5 \mathrm{wt} \%$, was found to have the longest $\mathrm{T} 1$ relaxation times. Conclusions: A patient-specific 3D-printed breast phantom was successfully designed and constructed using silicone and peanut oils to simulate the MR imaging characteristics of fibroglandular and adipose tissues. The phantom can be used to investigate different MR breast imaging protocols for the quantitative assessment of breast density.

Keywords: Magnetic resonance imaging (MRI); T1 and T2 relaxation times; fibroglandular-tissue; breast density; 3D-printing; fused deposition modelling (FDM); digital light processing (DLP); polylactic acid (PLA); photopolymer resin; silicone oil; peanut oil

Submitted Feb 12, 2020. Accepted for publication Apr 22, 2020.

doi: 10.21037/qims-20-251

View this article at: http://dx.doi.org/10.21037/qims-20-251

\section{Introduction}

Breast magnetic resonance imaging (MRI) is a wellestablished approach in the diagnosis of breast disease, and it has become an important modality in the screening of women at high-risk of breast cancer, preoperative staging of newly diagnosed breast cancer, and follow-up of breast cancer treatment (1-3). Hence, the European Society of Breast Imaging (EUSOBI) has recommended that breast 
MRI be used as an adjuvant modality in women at highrisk of developing breast cancer (3), for those with (BRCApositive genetic mutation carriers), family history, and/or high breast density (4).

Breast density, a measure of fibroglandular, dense tissue relative to fatty, non-dense tissue, is an independent risk factor of breast cancer (5-7). Consistent with this risk relationship, women who have dense breasts have a likelihood of developing breast cancer that is fourfold higher than those with fatty breasts $(8,9)$. Currently various methods have been developed and introduced to segment/ measure breast density using MRI: the utilization of a clustering algorithm, the segmentation of glandular and fatty tissues with an interactive thresholding algorithm, a logistic function approach and a curve-fitting algorithm; each has its advantages and limitations (10-14). However, there are certain drawbacks associated with the use of these algorithms as most of them are interpreted as measurements with a semi-automatic thresholding and segmentation methods. Likewise, different MR breastimaging protocols have been used to differentiate between adipose and fibroglandular tissues ranging from noncontrast-enhanced T1-weighted to contrast-enhanced T1weighted and diffusion-weighted acquisitions (15-19). Despite improvements in the quantitative assessment of breast density using MR imaging, there has been no general agreement about the optimal scanning protocol in this aspect. A recent systematic review and meta-analysis about the quantitative assessment of breast density has confirmed these variations among breast segmentation/measurement methods and MR breast-imaging protocols (20).

In recent years, there has been an increasing interest in 3D printing techniques, which are being used in different medical domains such as cardiovascular disease, orthopedic surgery, prosthetics, and neurosurgery (21-24). 3D-printed models have been shown to assist in the development of many surgical implants, which can improve the individual's understanding of such a complex anatomical structure (21). Several studies have produced anthropomorphic breast phantoms for X-ray imaging, but there is still insufficient data available for MR imaging (25-30). Carton et al. developed a $3 \mathrm{D}$ anthropomorphic breast phantom for the evaluation of image quality of $2 \mathrm{D}$ and $3 \mathrm{D}$ breast $\mathrm{X}$-ray imaging systems. This phantom was based on a computational model and a rapid prototyping technique to generate breast phantom with different compositions, sizes, and shapes by using a tissue-equivalent material (25). While the phantom has effectively demonstrated a heterogeneous distribution of the fibroglandular and adipose tissues that can be analogous to the clinical breast images, it has certain limitations in terms of its fabrication method and application. The phantom has been printed in slabs form, which is very complicated to manufacture and it is a timeconsuming process (25).

Although some research has been carried out on the use of $3 \mathrm{D}$ printing techniques to develop a breast phantom for MR imaging, only few studies have attempted to generate a personalized 3D-printed breast phantom based on a realistic breast MR images that can be similar to the anatomical structures seen in human tissues (26-30). Burfeindt et al. (26) reported a new and convenient synthetic procedure to develop an MRI-derived 3D-printed breast phantom for the preclinical use in microwave breast-imaging experiments. Although the phantom has successfully simulated the dielectric properties of the biological breast tissues, it has been designed for microwave breast-imaging rather than for MR imaging system (26). Furthermore, the importance of realistic phantom structure in the assessment of photoacoustic breast imaging systems for the purpose of simulating the acoustic and optical breast tissues properties was demonstrated in a study by Dantuma et al. (27), in which a semi-anthropomorphic 3D-printed moulds derived from a MRI segmented numerical breast model was developed to produce real breast morphology using polyvinyl chloride plastisol (PVCP). However, there are limits to how far the phantom that has been designed for ultrasound and photoacoustic imaging can be used to simulate the MR imaging characteristics of breast tissues (27). Moreover, He et al. (28) developed a 3D-printed breast phantom for machine calibration and image optimization in multi-modalities imaging, where a mixture of PVC powder and softener (i.e., dioctyl terephthalate) was used as a tissuemimicking material (TMM) of breast tissues. Although the study has successful demonstrated the simulation of breast structures, it has certain limitations in terms of the lack of the appearance, variability, and heterogeneity of structures that are presented in the physiological tissues (28). Another potential limitation is that the $\mathrm{T} 1$ and $\mathrm{T} 2$ relaxation times of the materials were measured and found to be shorter than those reported in the physiological human breast tissues $(28,29)$. While most of the aforementioned phantoms address their objectives in the medical imaging discipline, there are currently no phantoms available to evaluate the breast density based on a realistic morphology of breast structures derived from a MR images of human tissues. Likewise, uncertainties still exist about the most 


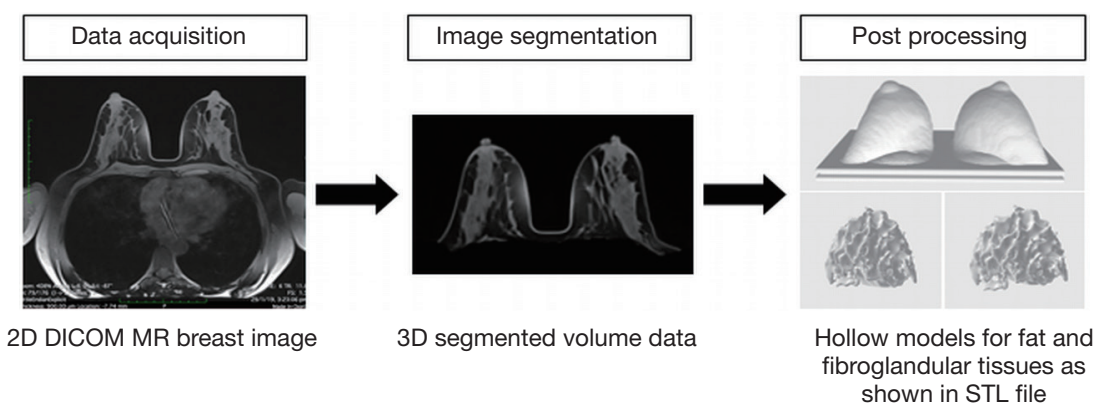

Figure 1 Schematic flowchart demonstrates the process of developing a patient-specific 3D-printed breast model.

appropriate TMMs that can be used to simulate the MRrelated characteristics and appearance of breast structures, particularly fibroglandular tissue. Such a personalized 3D-printed breast model could be used to examine different MR breast-imaging protocols not only to evaluate the breast density but also to determine the impact of applying various image quality parameters on the segmentation/ measurement methods of breast density. Therefore, the aim of this study is to develop a patient-specific 3D-printed breast phantom and to identify the most appropriate materials for simulating the MR imaging characteristics of fibroglandular and adipose tissues.

\section{Methods}

\section{Patient data}

Ethical approval was obtained from Curtin University's Human Research Ethics Committee (HREC) and King Fahd Armed Forces Hospital's (Jeddah) Research and Ethics Committee. A random sample of patients with no history of breast disease was retrospectively reviewed from an existing breast MRI database. The criteria for selecting the subjects were the following: no previous surgery, no radiotherapy treatment on the chest wall, no history of breast cancer, and a Breast Imaging-Reporting and Data System (BIRADS) classification of 1 , indicating a negative likelihood of cancer. A 46-year-old woman was identified by a senior radiology resident to match the selection criteria. The breast MRI examination was performed using $1.5 \mathrm{~T}$ system (MAGNETOM Aera, Siemens, Germany) with a dedicated breast coil (18 channels). The MR breast imaging protocol was chosen based on the recommendations of a recent systematic review and meta-analysis (20) as high-resolution non-contrast-enhanced T1-weighted images to allow a precise differentiation between adipose and fibroglandular tissues: TR/TE 11.8/6.0 ms; matrix size $384 \times 384$; slice thickness $0.9 \mathrm{~mm}$ with no gap.

\section{Image post-processing and segmentation process}

A series of image post-processing and segmentation of the volumetric data was performed. First, the anonymized Digital Imaging and Communications in Medicine (DICOM) MR images were imported into the commercially available software Analyze 12.0 (AnalyzeDirect, Inc., Lexana, KS, USA) to segment the non-contrast-enhanced T1-weighted breast images. Second, the breast's boundary was delineated manually to distinguish the breast's body from the surrounding tissues (pectoral muscle, heart, lungs, and thorax) on each 2D slice based on grayscale intensity, displayed in a histogram. Then, the $3 \mathrm{D}$ breast volume was created by these $2 \mathrm{D}$ images and was subsequently used to design the 3D-printed breast model. Finally, the 3D segmented MR breast volume was saved as a standard tessellation language (STL) file for further image postprocessing and 3D printing. Figure 1 presents a schematic flowchart of the process of developing a patient-specific 3D-printed breast model using MRI data. For more details, the phantom consists of three main parts: the outer shell, simulating the skin layer, and the internal structures, which include fibroglandular and fat tissues, imitating the breast composition. To generate the skin shell, the STL file containing the $3 \mathrm{D}$ segmented MR breast volume was imported into the Blender software, version 2.79b (Blender Foundation, Amsterdam, Netherlands) to hollow the model and ensure that all the internal structures were perfectly extracted.

On the other hand, the DICOM MR breast dataset was loaded into the 3D Slicer software, version 4.10.2 [National Alliance for Medical Image Computing (NA-MIC)] to segment out the fibroglandular tissue and ensure that all 

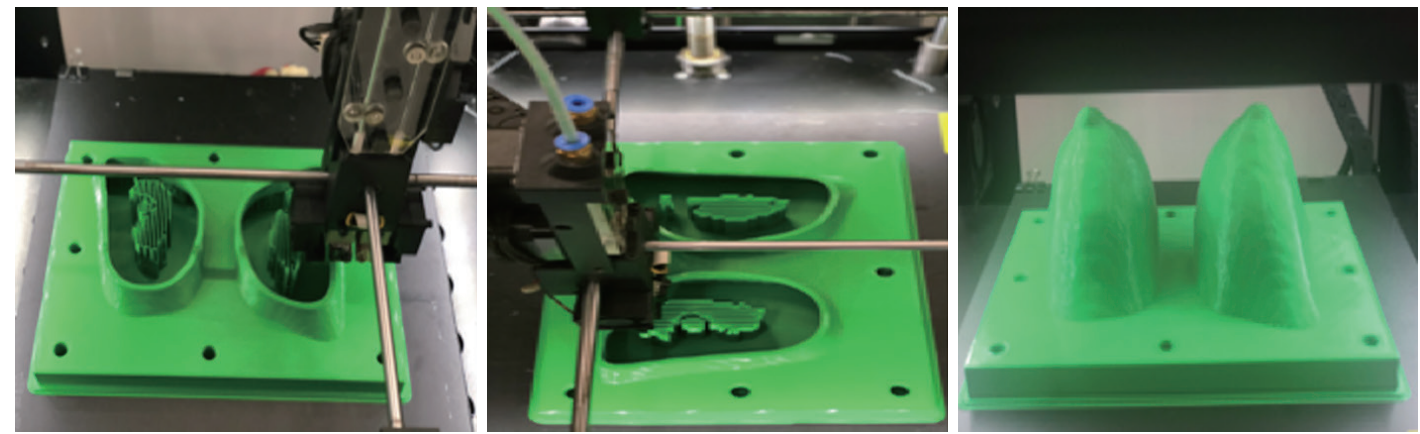

Figure 2 External structure of the patient-specific 3D-printed breast phantom that consists of 3 mm thick skin layer and compartments to be filled with fibroglandular and adipose tissues models.

the surrounding structures were completely removed. To increase reliability of the segmentation, each slice was segmented in different orientations using the threshold function, which was adjusted manually. This approach was used to threshold the DICOM dataset so that only the fibroglandular tissue structures were kept in the final segmented data. Subsequently, the segmented fibroglandular model was saved as a STL file and imported into the (version 3.5.474, Autodesk Inc., San Rafael, CA, USA) open-source software for further edit. Any deformities or free-floating objects were removed, and any holes were fixed during the editing process.

\section{Overview and breast phantom design}

This part is divided into three sections, each detailing the construction process related to the $3 \mathrm{D}$-printed breast model components.

\section{Skin layer}

Based on the dimensions of a realistic tissue, the outer phantom shell had an average thickness of $3.0 \mathrm{~mm}$, which corresponds to the normal skin thickness. The cover of the skin shell was designed using a computer-aided design (CAD) software. The skin shell and the cover were fabricated with fused deposition modelling (FDM) technology using polylactic acid (PLA) (Polymaker, Shanghai, China) on a Raise3D N2 Plus 3D printer (Raise3D, Irvine, CA, USA). The skin shell was printed with a layer height of $0.15 \mathrm{~mm}$, average printing time of 40 hours, and a resolution of $12.5 \mu \mathrm{m}$ (Figure 2).

\section{Fibroglandular region}

The fibroglandular models constitute the internal component of the $3 \mathrm{D}$-printed breast phantom. While various definitions of the term "breast density" have been proposed, in this study, the term "fibroglandular tissue" is used to refer to the breast density. Naturally, the fibroglandular region contains variable shapes and/or volumes of glandular tissue, includes fibrous or connective tissue. In clinical practice, the evaluation of fibroglandular tissue is based on a subjective assessment recommended by the American College of Radiology (ACR) BI-RADS, which is commonly used for mammography but also for MRI. The BI-RADS atlas can be described as a classification system that characterises breast density on the basis of the amount of fibroglandular tissue into four categories: (I) almost entirely fat, (II) scattered fibroglandular tissue, (III) heterogeneous fibroglandular tissue, and (IV) extreme fibroglandular tissue $(31,32)$.

In order to simulate the MR imaging characteristics, the $3 \mathrm{D}$ fibroglandular models were designed as hollow structures with an average thickness of $2.0 \mathrm{~mm}$. The fibroglandular models were fabricated using the digital light processing (DLP) technology on an Anycubic Photon S 3D DLP UV resin printer (Shenzhen Anycubic Technology Co. Ltd., Shenzhen, China) using white photopolymer resin (Magma H LINE Photopolymer Resin) from Magma Filament, Malaysia. A curing time of $10 \mathrm{sec}$ per layer, a layer thickness of $0.05 \mathrm{~mm}$, and a resolution of $47 \mu \mathrm{m}$ were used to fabricate the fibroglandular models. The printing duration for both left and right fibroglandular models was about 17 hours (Figure 3).

\section{Fat/adipose region}

This region comprises a considerable part of the $3 \mathrm{D}$-printed breast model. It consists of a selected material that simulates the MR imaging relaxation times of adipose tissue. 

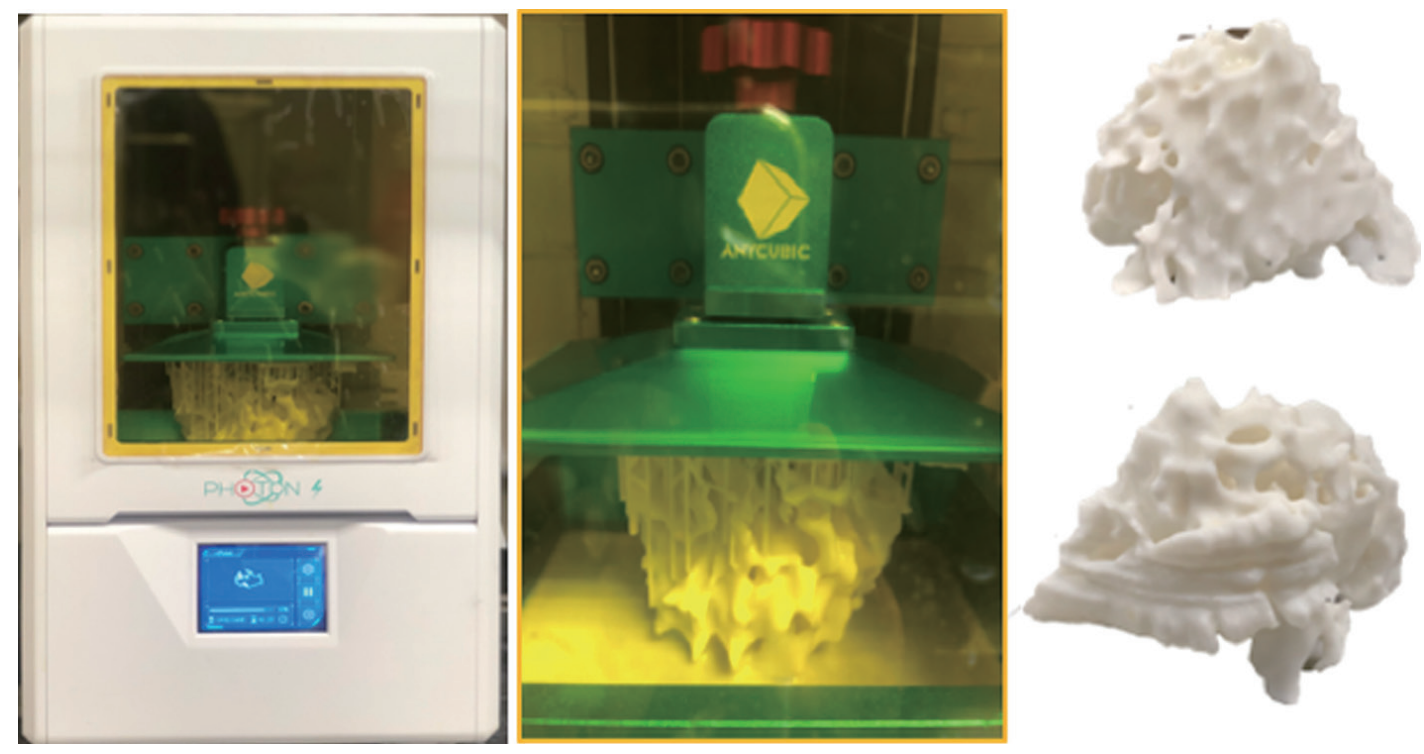

Figure 3 Fabrication of the hollow fibroglandular models using the Anycubic Photon S high-resolution 3D DLP UV resin printer. The thickness of the wall is $2.0 \mathrm{~mm}$. DLP, digital light processing.

\section{Fibroglandular TMMs}

Agarose gels with different concentrations vary in their ability to simulate the MR imaging characteristics of T1 and $\mathrm{T} 2$ relaxation times of an extensive range of human tissues $(33,34)$. In a study investigating the $\mathrm{T} 1$ and $\mathrm{T} 2$ relaxation times of four sample phantom liquids, Gach et al. (33) found that silicone oil had the longest T1 and T2 times on a $3 \mathrm{~T}$ MRI system: $1,068.29 \pm 5.95$ and $566.40 \pm 4.68 \mathrm{~ms}$, respectively. These results provide further support to the hypothesis that agarose gel or silicone oil could be used to mimic the MR imaging characteristics of fibroglandular tissue based on T1 and/or T2 relaxation times. Thus, four different raw materials were scanned to determine which one could be used to mimic the $\mathrm{T} 1$ and/or $\mathrm{T} 2$ relaxation times of fibroglandular tissue. The candidate materials were silicone oil with a viscosity of $50 \mathrm{~mm}^{2} / \mathrm{s}$ at $25^{\circ} \mathrm{C}$ (TEX Chemical Inc., Country), agarose (Thermo Fisher, Waltham, MA, USA), silicone rubber RTV (Craftiviti Sdn. Bhd., Selangor, Malaysia), and fish oil (Blackmores, Sydney, Australia) (Figure 4).

\section{Fat/adipose TMMs}

As Niebuhr et al. (35) reported, olive oil successfully simulates the MR imaging relaxation times of adipose tissue in abdominal and pelvic tissues measured in-vivo. In another study, Niebuhr et al. (34) found that peanut oil efficiently simulates the MR imaging characteristics of subcutaneous fat for pelvis phantoms. Peanut oil was preferred in this study for several reasons, including its relatively similar MR imaging characteristics ( $\mathrm{T} 1$ and $\mathrm{T} 2$ relaxation times) for adipose tissue, its translucent appearance, and its high oxidation stability $(34,35)$. These characteristics suggest that peanut oil could be a performed material to mimic the $\mathrm{T} 1 \mathrm{and} / \mathrm{or} \mathrm{T} 2$ relaxation times of breast adipose tissue. Two types of peanut oil were scanned for testing: peanut oil Basso (raw material: Arachis hypogea; price: US\$ 5/1L; Basso), and peanut oil Pressed Purity (raw materials: oleic acid (96.2\%) and linoleic acid (13.2\%), price: US\$18/1.5L, Proteco Oils) (Figure 4).

\section{Breast phantom construction}

The $\mathrm{T} 1$ relaxation times of the five selected materials (agarose gel, silicone rubber with/without fish oil, silicone oil, and peanut oil) were measured at room temperature using a 3 T MRI system to determine which ones could be used to mimic the MR imaging characteristics of fibroglandular and adipose tissues. The results were then compared to the reference values of $\mathrm{T} 1$ relaxation times of the corresponding tissues. Following this, the materials that matched the $\mathrm{T} 1$ relaxation times of the respective tissues were chosen to fill the 3D-printed hollow breast shells. 

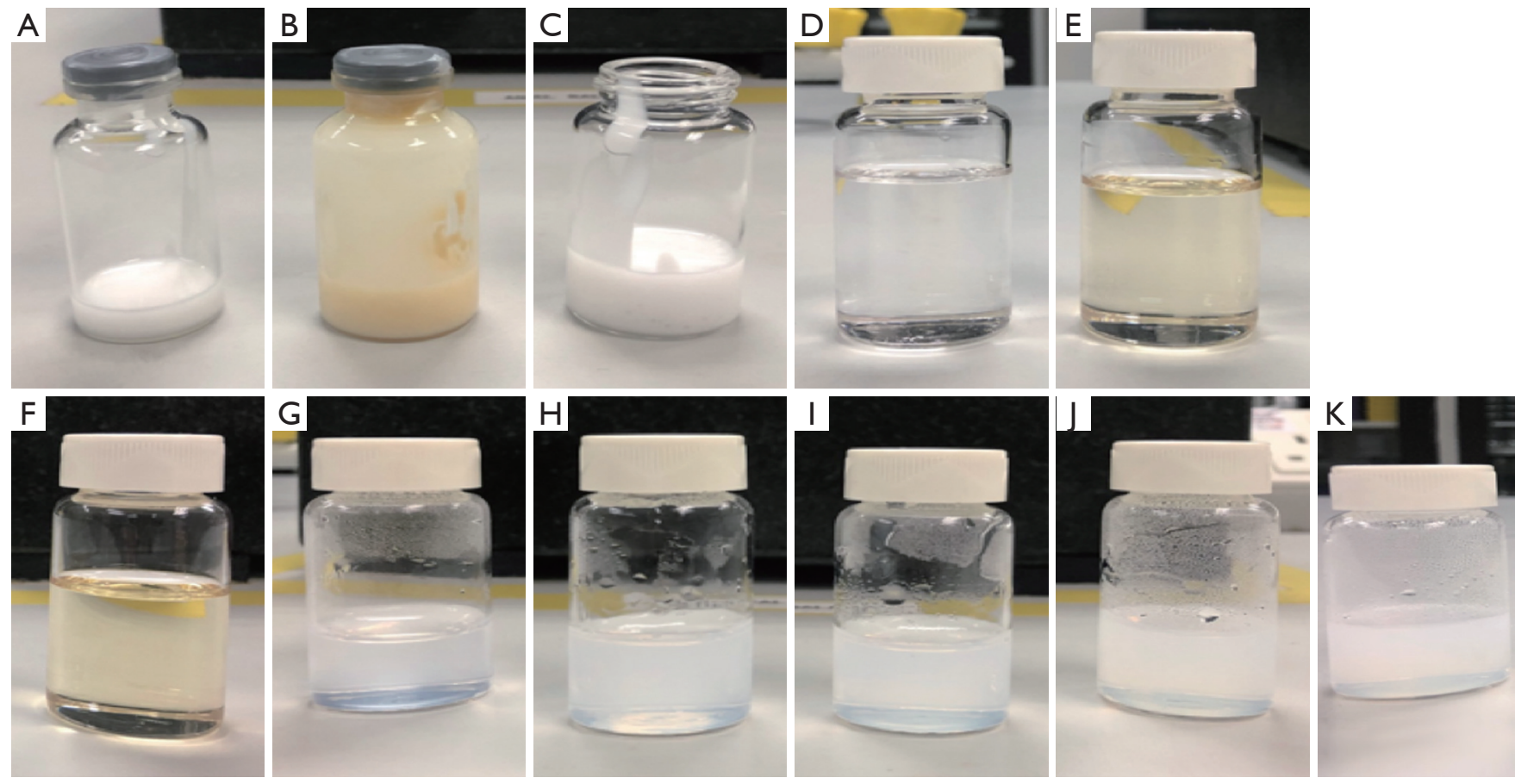

Figure 4 Test raw materials. (A) Silicone rubber; (B) silicone rubber with fish oil; (C) fresh silicone rubber; (D) silicone oil with a viscosity of $50 \mathrm{~mm}^{2} / \mathrm{s}$; (E) peanut oil (Basoo); (F) peanut oil (pressed purity); (G) agarose gel $0.5 \mathrm{wt} \%$; (H) agarose gel $1.0 \mathrm{wt} \%$; (I) agarose gel 1.5 wt\%; (J) agarose gel $2.0 \mathrm{wt} \%$; (K) agarose gel $2.5 \mathrm{wt} \%$.

\section{Results}

\section{D-printed hollow models}

The 3D-printed models of the hollow skin and fibroglandular region shells were scanned on a $3 \mathrm{~T}$ MRI system (MAGNETOM Prisma, Siemens Healthcare, Erlangen, Germany) to check whether the models printed with the PLA or the photopolymer resin produce MR signals corresponding with these tissue features. Fortunately, no MR signal was observed from scanning the $3 \mathrm{D}$-printed hollow models, indicating the possibility of using these materials for breast structure simulation and further patient models. It is important to note that the selected materials were checked when the $3 \mathrm{D}$ printing was initially performed and then checked again at the end of the breast phantom construction.

\section{Sample characteristics}

The five selected materials were scanned on the same 3T MRI system, with the materials placed in the 18-channel body and 32-channel spine coils. The MR breast scanning was chosen based on the institutional clinical protocol using
3D T1- and T2-weighted turbo spin echo (TSE) sequences: TR/TE 650.0/10.0 ms; matrix size 384×384; slice thickness $2.9 \mathrm{~mm}$; no gap, and TR/TE 6,080.0/78.0 ms; matrix size $384 \times 384$; slice thickness $4.0 \mathrm{~mm}$; no gap, respectively.

Figure 5 presents the MR imaging T1 relaxation times of the five materials (agarose gel, silicone rubber with/without fish oil, silicone oil, and peanut oil) simulating the breast composition. Figure $5 D$ shows that the T1-weighted image of the silicone oil was associated with a mid-grey signal intensity, which is usually related to water-based tissues characterized by a moderate $\mathrm{T} 1$ relaxation time. On the other hand, Figure 5E,F shows that the T1-weighted images of the peanut oils indicated a high signal intensity, which was within expectation, as fat-based tissues have a short $\mathrm{T} 1$ relaxation time. In contrast, the T1-weighted images of the agarose gel with different concentrations, 0.5 to $2.5 \mathrm{wt} \%$; were associated with low signal intensity, which is mainly observed in free water and other fluids (Figure $5 G, H, I, \mathcal{F}, K$ ).

\section{T1 relaxation times of the sample materials}

The $\mathrm{T} 1$ relaxation times of these five materials are listed in Table 1. Silicone oil had a T1 relaxation time similar to 

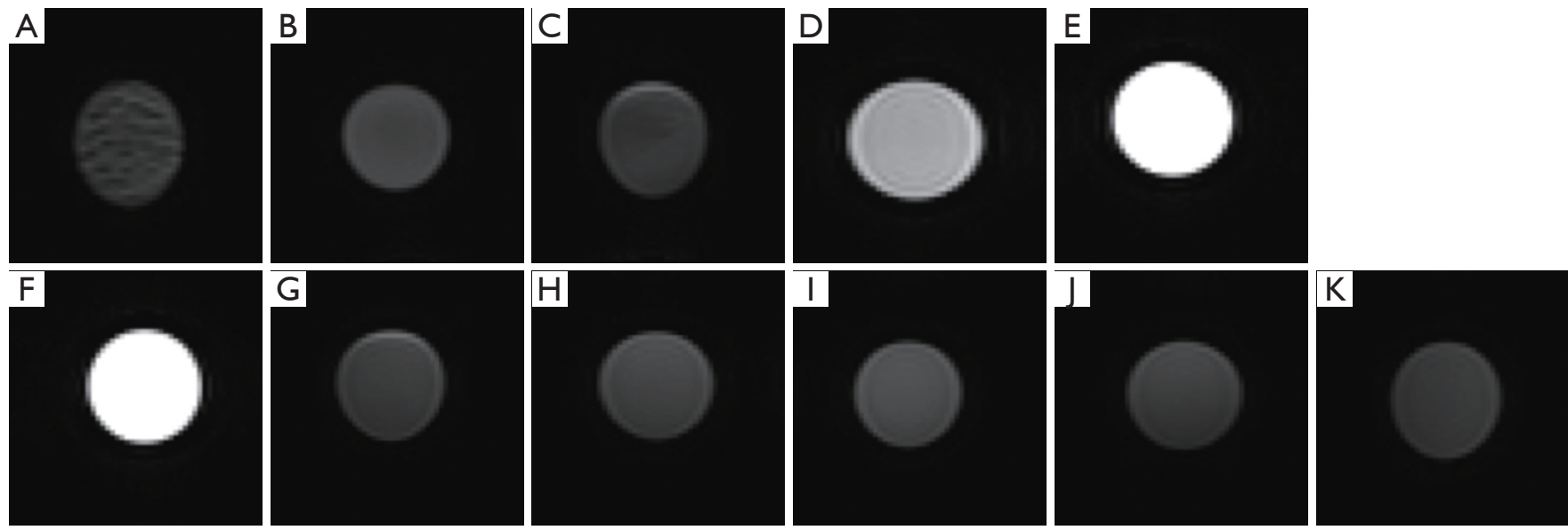

Figure 5 T1-weighted images. (A) Silicone rubber; (B) silicone rubber with fish oil; (C) fresh silicone rubber; (D) silicone oil with a viscosity of $50 \mathrm{~mm} / \mathrm{s}$; (E) peanut oil (Basoo); (F) peanut oil (Pressed Purity); (G) agarose gel $0.5 \mathrm{wt} \%$; (H) agarose gel 1.0 wt\%; (I) agarose gel 1.5 $\mathrm{wt} \%$; (J) agarose gel $2.0 \mathrm{wt} \%$; (K) agarose gel $2.5 \mathrm{wt} \%$.

Table 1 T1 Relaxation times of different materials for tissue surrogates used in the experiment

\begin{tabular}{lc}
\hline Phantom, TMM & $\begin{array}{c}\text { T1 (average SD, ms), } \\
\text { 3T Siemens MR Scanner }\end{array}$ \\
\hline Fibroglandular shell & No signal \\
Skin/outer shell & No signal \\
Silicone rubber & $577.2 \pm 107.8$ \\
Silicone rubber with fish oil & $902.1 \pm 120.5$ \\
Fresh silicone rubber & $638.3 \pm 108.5$ \\
Silicone oil $50 \mathrm{~mm}^{2} / \mathrm{s}^{*}$ & $1,515.8 \pm 105.5$ \\
Peanut oil (Basso) & $405.4 \pm 15.1$ \\
Peanut oil (pressed purity) & $404.1 \pm 10.5$ \\
Agarose gel $0.5 \mathrm{wt} \%$ & $4015.5 \pm 100.2$ \\
Agarose gel $1.0 \mathrm{wt} \%$ & $3,877.8 \pm 130.5$ \\
Agarose gel $1.5 \mathrm{wt} \%$ & $3,404.8 \pm 255.9$ \\
Agarose gel $2.0 \mathrm{wt} \%$ & $3,572.6 \pm 100.4$ \\
Agarose gel $2.5 \mathrm{wt} \%$ & $3,617.2 \pm 101.5$ \\
\hline
\end{tabular}

*, Viscosity unit. TMM, tissue-mimicking material.

that of fibroglandular tissue: $1,515.8 \pm 105.5 \mathrm{~ms}$. In contrast, the Basso and Pressed Purity peanut oils had T1 relaxation times analogous to that of adipose tissue: $405.4 \pm 15.1$ and $404.1 \pm 10.5 \mathrm{~ms}$, respectively. For comparison, the T1 and $\mathrm{T} 2$ relaxation times of fibroglandular and adipose tissues measured using a $1.5 \mathrm{~T}$ and a $3 \mathrm{~T}$ MRI system are presented in Table 2.

As shown in Table 1, the agarose gel with different concentrations, ranging from 0.5 to $2.5 \mathrm{wt} \%$, had the longest $\mathrm{T} 1$ relaxation times, which are similar to that of free water. The interesting finding is that the lowest concentration was associated with the highest $\mathrm{T} 1$ relaxation time. Overall, the results presented in Table 1 and Figure 5 indicate that the silicone and peanut oils closely resemble the MR imaging T1 relaxation times of the fibroglandular and adipose tissues, respectively. Therefore, these materials were chosen to fill the $3 \mathrm{D}$-printed hollow models.

Figure 6 provides an overview of the construction process of the $3 \mathrm{D}$-printed breast phantom. The two fibroglandular shell models were filled with a silicone oil and then sealed using UV-curable photopolymer resin. Following this, the filled fibroglandular shell models were fixed inside the skin shell model using acrylic-based adhesive. Further, the space between the fibroglandular and skin shell models was filled with peanut oil. A home-made silicone gasket and cover were used to enclose the breast phantom. Finally, the cover was tighten using the commercially available polycarbonate bolt and nuts.

\section{Scanning of the 3D-printed breast phantom}

The MR images of the phantom were acquired following the same breast imaging protocols described in the Results, Sample Characteristics. The phantom was scanned in a 
Table $2 \mathrm{~T} 1$ and T2 Relaxation times of the breast tissues at 1.5T and 3T using FSE-IR scans (36)

\begin{tabular}{lcccc}
\hline Tissue (reference) & T1 (average SD, ms), & T2 (average SD, ms), & T1 (average SD, ms), 3T & T2 (average SD, ms), 3T \\
\hline Adipose/fat & $1.5 T$ & $1.5 T$ & $449.27 \pm 26.09$ & $52.96 \pm 1.54$ \\
Fibroglandular & $372.04 \pm 8.6$ & $53.33 \pm 2.11$ & $1,324.42 \pm 167.63$ & $54.36 \pm 9.35$ \\
\hline
\end{tabular}

FSE-IR, Fast Spin Echo-Inversion Recovery.

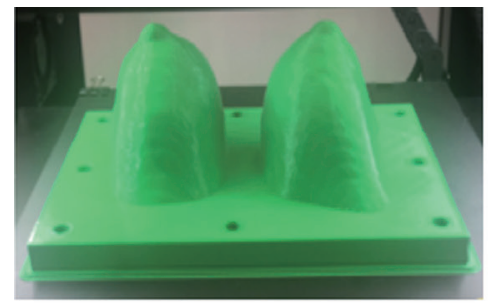

Skin/outer shell model
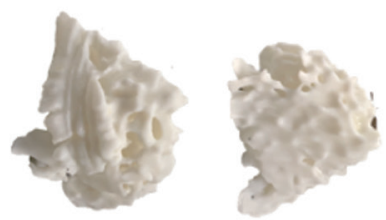

Fibroglandular shell models were filled with a silicone oil.
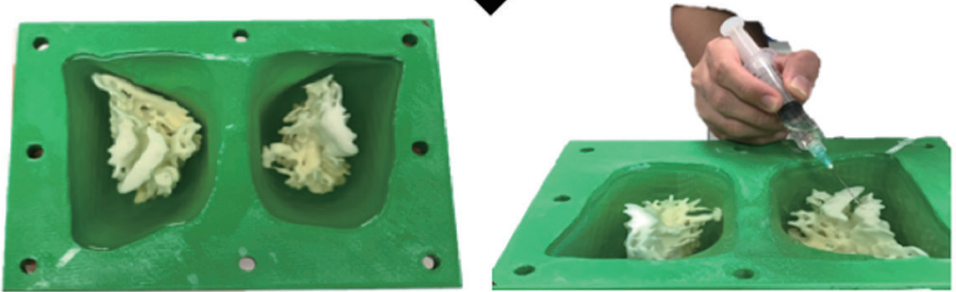

The fibroglandular models were attached to the skin shell model using acrylic-based adhesive. Then, the skin shell model was filled with a peanut oil.

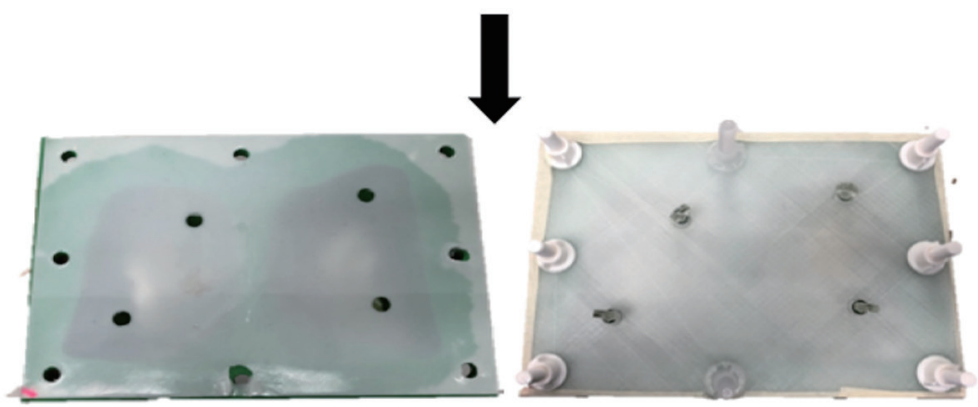

The peanut oil was enclosed in the breast phantom using a silicone gasket and cover. Finally, the cover was tightened up using polycarbonate bolt and nuts.

Figure 6 Flow chart showing 3D construction of the breast phantom. 3D printing technique was used to create the hollow shells for skin and fibroglandular regions. Fibroglandular and adipose tissues were simulated using silicone and peanut oils, respectively.

prone position using a dedicated 18 -channel breast coil. Figure 7 shows the T1- and T2-weighted MR images of a patient-specific 3D-printed breast phantom using silicone and peanut oils as surrogates of the fibroglandular and adipose tissues, respectively. These oils presented an acceptable level of contrast and MR-related characteristics in both the T1- and the T2-weighted images. One of the most noticeable features of this phantom is that it is slightly inhomogeneous. However, this feature simulates the considerable inhomogeneity as often observed among 


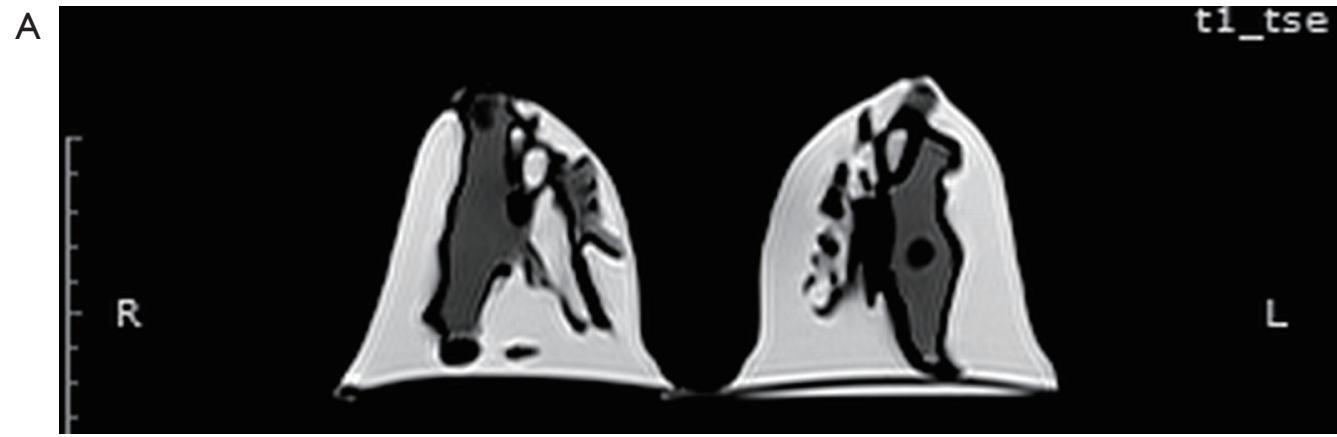

B

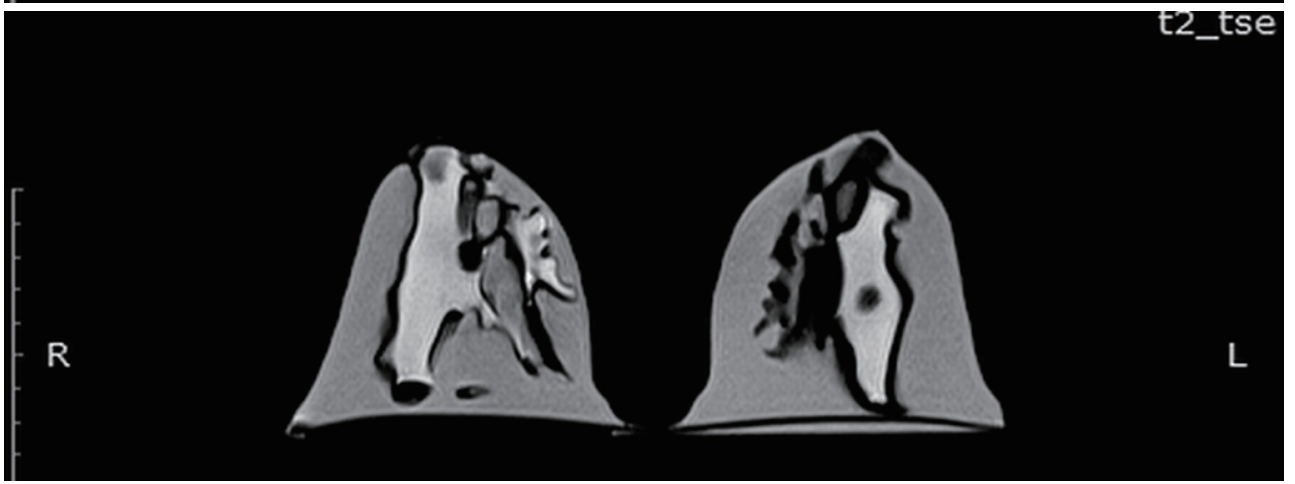

Figure $7 \mathrm{MR}$ images of the 3D printed breast phantom. (A) T1-weighted image; (B) T2-weighted image using TSE scans. TSE, turbo spin echo.

the irregular distribution of the patient. Overall, the results shown in Table 1 and Figure 5 indicate that the MR imaging $\mathrm{T} 1$ relaxation times of the silicone and peanut oils used for the simulation of fibroglandular and adipose tissues are similar to their respective reference values reported in the literature.

\section{Discussion}

This study aimed to develop a patient-specific 3D-printed breast phantom and to determine the most appropriate materials for simulating the MR imaging characteristics of fibroglandular and adipose tissues. Anthropomorphic shapes of skin and fibroglandular tissues were constructed using 3D-printing techniques based on the segmentations of breast MR images from a selected healthy patient's data. All the 3D skin and fibroglandular region shells were designed as hollow structures using PLA and photopolymer resin. Since no MR signal was generated by the $3 \mathrm{D}$-printed hollow models of those corresponding shells, different materials were selected to search for suitable ones with silicone oil and peanut oil being the most appropriate materials with similar T1 relaxation times to fibroglandular and adipose tissues.

It was assumed that the $\mathrm{T} 1$ relaxation times would effectively supplement and extend our knowledge about the selected materials since most organs' $\mathrm{T} 1$ values are five times longer than their T2 values. A comparison of the $\mathrm{T} 1$ relaxation times of the scanned materials with breast structure and literature reports showed that the silicone and peanut oils closely resemble the MR imaging T1 relaxation times of the fibroglandular and adipose tissues, respectively. Surprisingly, this study did not find a significant difference in the $\mathrm{T} 1$ relaxation times between different concentrations of agarose gel, which exhibited long T1 relaxation times, similar to that of water. Nevertheless, the agarose gel can be mixed with a gadolinium-based contrast agent for $\mathrm{T} 1$ adaptation, and can thus be used to simulate the MR imaging relaxation times of a wide range of human tissues. However, this would be costly and requires precautions when handling the contrast agent. Another unexpected finding was the slight difference in the T1 relaxation times between the Basso and Pressed Purity peanut oils. However, the observed difference was not significant. It is also worth noting that the Basso peanut oil was preferable due to its purity, availability, and low cost. 
The most important clinically relevant finding was that the silicone and peanut oils demonstrated an acceptable level of contrast and MR-related characteristics of breast structures in both the T1- and the T2-weighted images. These findings are in line with Niebuhr et al. (34), who suggested that peanut oil efficiently simulates the MR imaging characteristics of subcutaneous fat for pelvis phantoms. In accordance with the present results, previous studies demonstrated that silicone oil with a viscosity of $50 \mathrm{~mm}^{2} / \mathrm{s}$ had the longest $\mathrm{T} 1$ and $\mathrm{T} 2$ relaxation times on a $3 \mathrm{~T}$ MRI system $(33,35)$. However, silicone oil was not previously used for simulating the MRrelated characteristics of breast structures, particularly fibroglandular tissue. Thus, this study presents interesting findings to encourage more research along this direction in breast phantom.

The observed correlation between silicone oil's T1 relaxation time and fibroglandular tissue could be attributed to its chemical composition and physical properties such as viscosity and density. This preliminary finding; suggests that silicone and peanut oils can be used to efficiently simulate the MR imaging characteristics of breast structures and produce further models. An implication of this is the possibility to examine different MR breast imaging protocols to identify the most appropriate for the quantitative assessment of breast density. For future investigations, it might be possible to use different chemical compositions and physical properties of silicone oils to evaluate the MR imaging relaxation times of breast structures. Since the relationship between silicone oil and fibroglandular tissue has not been studied, further research is required to better understand it.

Although the study has successfully designed and constructed a patient-specific 3D-printed breast phantom, the findings are subject to several limitations. The study was not specifically designed to evaluate the mechanical properties of breast tissue components, such as elastic modulus or tissue strength. Examining the mechanical features along with the physical properties of selected materials could provide an idea of their characteristics and allow more detailed comparisons to the human breast tissue. Moreover, there are certain drawbacks to the use of $3 \mathrm{D}$ printing techniques for the construction of skin and fibroglandular hollow shells. One of them is the potential risk for some of the connected structures to break easily during the cleaning process. For this reason, several models of varying wall thicknesses, ranging between 1.0 and $2.5 \mathrm{~mm}$, were printed. However, increasing the thickness of photopolymer resin can cause considerable deformation of the fibroglandular structure. Another potential limitation is due to the complexity of the fibroglandular structure, with holes formed in the final mould. For this reason, a wrapping process was performed manually for each model to ensure that all the small gaps had been completely sealed.

A further study on a patient-specific 3D-printed breast phantom will be conducted with a focus on different percentages of fibroglandular tissue. This can correspond to the four categories of the ACR BI-RADS atlas, thus allowing an estimation of the volumes of fibroglandular tissue. Varying its proportions will allow the quantitative assessments of breast density to be performed.

\section{Conclusions}

In this study, a patient-specific $3 \mathrm{D}$-printed breast phantom was successfully constructed using silicone and peanut oils to simulate the MR-related characteristics of breast fibroglandular and adipose tissues. The proposed methodologies can be used as a preliminary work for breast structure simulations and the construction of further patient models using MRI dataset. The phantom can be used to test different breast MR imaging protocols to determine the optimum scanning parameters and analysis algorithms for the quantitative assessment of breast density.

\section{Acknowledgments}

We like to thank King Fahd Armed Forces Hospital, Jeddah, Kingdom of Saudi Arabia, Mrs. Aghadeer Bana, Medical Physicist, and Ms. Khulud Al-Amoudi, Radiology Technologist/Patient Experience Western Regional Head for their cooperation in ethical approval and data collection. We deeply acknowledge the support of Dr. Sarah Bukhari, Senior Radiology Resident for her expertise guidance in gathering the required healthy patient's data.

Funding: This project was funded by the Ministry of Defence, Medical Service Division, Kingdom of Saudi Arabia and Saudi Arabian Cultural Mission (SACM), Australia.

\section{Footnote}

Conflicts of Interest: All authors have completed the ICMJE uniform disclosure form (available at http://dx.doi. org/10.21037/qims-20-251). ZS serves as an unpaid associate editor of Quantitative Imaging in Medicine and Surgery. The 
other authors have no other conflicts of interest to declare.

Ethical Statement: Ethical approval was obtained from relevant institutions for use of MR images to generate patient-specific 3D printed model.

Open Access Statement: This is an Open Access article distributed in accordance with the Creative Commons Attribution-NonCommercial-NoDerivs 4.0 International License (CC BY-NC-ND 4.0), which permits the noncommercial replication and distribution of the article with the strict proviso that no changes or edits are made and the original work is properly cited (including links to both the formal publication through the relevant DOI and the license). See: https://creativecommons.org/licenses/by-nc-nd/4.0/.

\section{References}

1. Sardanelli F, Boetes C, Borisch B, Decker T, Federico M, Gilbert FJ, Helbich T, Heywang-Köbrunner SH, Kaiser WA, Kerin MJ, Mansel RE. Magnetic resonance imaging of the breast: recommendations from the EUSOMA working group. Eur J Cancer 2010;46:1296-316.

2. Londero V, Zuiani C, Linda A, Girometti R, Bazzocchi M, Sardanelli F. High-risk breast lesions at imaging-guided needle biopsy: usefulness of MRI for treatment decision. AJR Am J Roentgenol 2012;199:W240-50.

3. Mann RM, Balleyguier C, Baltzer PA, Bick U, Colin C, Cornford E, Evans A, Fallenberg E, Forrai G, Fuchsjäger MH, Gilbert FJ, Helbich TH, Heywang-Köbrunner SH, Camps-Herrero J, Kuhl CK, Martincich L, Pediconi F, Panizza P, Pina LJ, Pijnappel RM, Pinker-Domenig K3, Skaane P, Sardanelli F; European Society of Breast Imaging (EUSOBI), with language review by Europa Donna-The European Breast Cancer Coalition. Breast MRI: EUSOBI recommendations for women's information. Eur Radiol 2015;25:3669-78.

4. Heller SL, Yeong Lin LL, Melsaether AN, Moy L, Gao Y. Hormonal Effects on Breast Density, Fibroglandular Tissue, and Background Parenchymal Enhancement. Radiographics 2018;38:983-96.

5. Chen JH, Chang YC, Chang D, Wang YT, Nie K, Chang RF, Nalcioglu O, Huang CS, Su MY. Reduction of breast density following tamoxifen treatment evaluated by 3-D MRI: preliminary study. Magn Reson Imaging 2011;29:91-8.

6. Wang J, Azziz A, Fan B, Malkov S, Klifa C, Newitt D, Yitta S, Hylton N, Kerlikowske K, Shepherd JA.
Agreement of mammographic measures of volumetric breast density to MRI. PLoS One 2013;8:e81653.

7. Tagliafico A, Bignotti B, Tagliafico G, Astengo D, Martino L, Airaldi S, Signori A, Sormani MP, Houssami N, Calabrese M. Breast density assessment using a 3T MRI system: Comparison among different sequences. PLoS One 2014;9:e99027.

8. Lienart V, Carly B, Kang X, Guzy L, Sajovitz AM, Liebens F. Effect of preventive hormonal therapy on breast density: a systematic qualitative review. ScientificWorldJournal 2014;2014:942386.

9. Tagliafico A, Tagliafico G, Astengo D, Airaldi S, Calabrese $M$, Houssami N. Comparative estimation of percentage breast tissue density for digital mammography, digital breast tomosynthesis, and magnetic resonance imaging. Breast Cancer Res Treat 2013;138:311-7.

10. Nayeem F, Ju H, Brunder DG, Nagamani M, Anderson KE, Khamapirad T, Lu LJ. Similarity of fibroglandular breast tissue content measured from magnetic resonance and mammographic images and by a mathematical algorithm. Int J Breast Cancer 2014;2014:961679.

11. Lin M, Chan S, Chen JH, Chang D, Nie K, Chen ST, Lin CJ, Shih TC, Nalcioglu O, Su MY. A new bias field correction method combining $\mathrm{N} 3$ and FCM for improved segmentation of breast density on MRI. Med Phys 2011;38:5-14.

12. Doran SJ, Hipwell JH, Denholm R, Eiben B, Busana M, Hawkes DJ, Leach MO, Silva ID. Breast MRI segmentation for density estimation: Do different methods give the same results and how much do differences matter? Med Phys 2017;44:4573-92.

13. Tagliafico A, Tagliafico G, Tosto S, Chiesa F, Martinoli C, Derchi LE, Calabrese M. Mammographic density estimation: comparison among BI-RADS categories, a semi-automated software and a fully automated one. Breast 2009;18:35-40.

14. Boston RC, Schnall MD, Englander SA, Landis JR, Moate PJ. Estimation of the content of fat and parenchyma in breast tissue using MRI T1 histograms and phantoms. Magn Reson Imaging 2005;23:591-9.

15. Klifa C, Carballido-Gamio J, Wilmes L, Laprie A, Shepherd J, Gibbs J, Fan B, Noworolski S, Hylton N. Magnetic resonance imaging for secondary assessment of breast density in a high-risk cohort. Magn Reson Imaging 2010;28:8-15.

16. Chang DH, Chen JH, Lin M, Bahri S, Yu HJ, Mehta RS, Nie K, Hsiang DJ, Nalcioglu O, Su MY. Comparison of breast density measured on MR images acquired using fat- 
suppressed versus non-fat-suppressed sequences. Med Phys 2011;38:5961-68.

17. Choi YJ, Chen JH, Yu HJ, Li Y, Su MY. Impact of Different Analytic Approaches on the Analysis of the Breast Fibroglandular Tissue Using Diffusion Weighted Imaging. Biomed Res Int 2017;2017:1094354.

18. McDonald ES, Schopp JG, Peacock S, DeMartini WB, Rahbar H, Lehman CD, Partridge SC. Diffusion-weighted MRI: association between patient characteristics and apparent diffusion coefficients of normal breast fibroglandular tissue at 3 T. AJR Am J Roentgenol 2014;202:W496-502.

19. Ledger AE, Scurr ED, Hughes J, Macdonald A, Wallace T, Thomas K, Wilson R, Leach MO, Schmidt MA. Comparison of Dixon sequences for estimation of percent breast fibroglandular tissue. PLoS One 2016;11:e0152152.

20. Sindi R, Sá Dos Reis C, Bennett C, Stevenson G, Sun Z. Quantitative Measurements of Breast Density Using Magnetic Resonance Imaging: A Systematic Review and Meta-Analysis. J Clin Med 2019. doi: 10.3390/jcm8050745.

21. Matsumoto JS, Morris JM, Foley TA, Williamson EE, Leng S, McGee KP, Kuhlmann JL, Nesberg LE, Vrtiska TJ. Threedimensional physical modeling: applications and experience at Mayo Clinic. Radiographics 2015;35:1989-2006.

22. Sun Z, Lau I, Wong YH, Yeong CH. Personalized threedimensional printed models in congenital heart disease. J Clin Med 2019. doi: 10.3390/jcm8040522.

23. Perica ER, Sun Z. A Systematic Review of ThreeDimensional Printing in Liver Disease. J Digit Imaging 2018;31:692-701.

24. Sun Z, Liu D. A systematic review of clinical value of three-dimensional printing in renal disease. Quant Imaging Med Surg 2018;8:311-25.

25. Carton AK, Bakic P, Ullberg C, Derand H, Maidment AD. Development of a physical 3D anthropomorphic breast phantom. Med Phys 2011;38:891-6.

26. Burfeindt MJ, Colgan TJ, Mays RO, Shea JD, Behdad N, Van Veen BD, Hagness SC. MRI-Derived 3-D-Printed Breast Phantom for Microwave Breast Imaging Validation. IEEE Antennas Wirel Propag Lett 2012;11:1610-3.

27. Dantuma M, van Dommelen R, Manohar S. Semianthropomorphic photoacoustic breast phantom. Biomed Opt Express 2019;10:5921-39.

28. He Y, Liu Y, Dyer BA, Boone JM, Liu S, Chen T, Zheng F, Zhu Y, Sun Y, Rong Y, Qiu J. 3D printed breast phantom for multi-purpose and multi-modality imaging. Quant Imaging Med Surg 2019;9:63-74.

29. He Y, Qin S, Dyer BA, Zhang H, Zhao L, Chen T, Zheng F, Sun Y, Shi L, Rong Y, Qiu J. Characterizing mechanical and medical imaging properties of polyvinyl chloridebased tissue-mimicking materials. J Appl Clin Med Phys 2019;20:176-83.

30. Freed M, de Zwart JA, Loud JT, El Khouli RH, Myers KJ, Greene MH, Duyn JH, Badano A. An anthropomorphic phantom for quantitative evaluation of breast MRI. Med Phys 2011;38:743-53.

31. American College of Radiology. ACR BI-RADS® Atlas: Breast Imaging Reporting and Data System. Reston: American College of Radiology, 2013.

32. Mainiero MB, Lourenco A, Mahoney MC, Newell MS, Bailey L, Barke LD, D'Orsi C, Harvey JA, Hayes MK, Huynh PT, Jokich PM, Lee SJ, Lehman CD, Mankoff DA, Nepute JA, Patel SB, Reynolds HE, Sutherland ML, Haffty BG. ACR Appropriateness Criteria Breast Cancer Screening. J Am Coll Radiol 2016;13:R45-9.

33. Gach HM. T1 and T2 and complex permittivities of mineral oil, silicone oil, and glycerol at $0.35,1.5$, and $3 \mathrm{~T}$. Med Phys 2019;46:1785-92.

34. Niebuhr NI, Johnen W, Echner G, Runz A, Bach M, Stoll M, Giske K, Greilich S, Pfaffenberger A. The ADAM-pelvis phantom-an anthropomorphic, deformable and multimodal phantom for MRgRT. Phys Med Biol 2019;64:04NT05.

35. Niebuhr NI, Johnen W, Güldaglar T, Runz A, Echner G, Mann P, Möhler C, Pfaffenberger A, Jäkel O, Greilich $\mathrm{S}$. Radiological properties of tissue surrogates used in a multimodality deformable pelvic phantom for MR-guided radiotherapy. Med Phys 2016;43:908-16.

36. Rakow-Penner R, Daniel B, Yu H, Sawyer-Glover A, Glover GH. Relaxation times of breast tissue at $1.5 \mathrm{~T}$ and $3 \mathrm{~T}$ measured using IDEAL. J Magn Reson Imaging 2006;23:87-91.

Cite this article as: Sindi R, Wong $\mathrm{YH}$, Yeong $\mathrm{CH}$, Sun $\mathrm{Z}$ Development of patient-specific 3D-printed breast phantom using silicone and peanut oils for magnetic resonance imaging. Quant Imaging Med Surg 2020;10(6):1237-1248. doi: 10.21037/qims20-251 